\title{
The ATLAS public website - Evolution to Drupal 8
}

\author{
Meirin Oan Evans ${ }^{a, *}$, on behalf of the ATLAS Collaboration \\ ${ }^{a}$ University of Sussex, \\ Falmer, Brighton, Great Britain \\ E-mail: meirin. oan. evans@cern.ch
}

Four years after the deployment of the ATLAS public website using the Drupal 7 content management system, the ATLAS Education \& Outreach group is in the process of migrating to the new CERN Drupal 8 infrastructure. We present lessons learned from the development, usage and evolution of the original web site, and how the choice of technology helped to shape and reinforce our communication strategy. We then discuss tactics for the migration to Drupal 8, including our choice to use the CERN Override theme. This theme was developed by the CERN web team to support clients like ATLAS to develop web sites in the relatively complex and nonintuitive environment of Drupal. Furthermore, CERN has encouraged usage of this theme to ease support and future migration. We present the effects this choice has on the design, implementation, operation and maintenance of the new site.

40th International Conference on High Energy physics - ICHEP2020

July 28 - August 6, 2020

Prague, Czech Republic (virtual meeting) 


\section{Motivation}

Communication of scientific goals, progress and achievements is a basic necessity of all fundamental research. This is particularly true for the large, publicly-funded international collaborations of the Large Hadron Collider (LHC) at CERN [1]. Not only does the public have the right to know how and toward what aim their resources are being spent, but they are key stakeholders of the knowledge that is being gained.

Beyond the social responsibility of scientific communication, however, lies its ability to reach world-wide audiences with messages that go above and beyond the reporting of results. These can include the tools of science and the methodology of the scientific process, the value of international collaboration, and the contributions being made to health, finance, security and other indirectly related fields. The ATLAS Collaboration takes advantage of its communication platforms to bring these messages to a variety of global audiences, thus securing support for the field through key stakeholders and decision makers, and sparking interest in the broader public and the next generation of scientists and engineers.

The primary platform supporting this communication is the ATLAS public website [2]], as shown in Figure 1. The ATLAS public website gives access to:

- Descriptions of the ATLAS Experiment [3] and its collaboration.

- Multimedia educational resources for teachers, collaboration members, media and others.

- Updates (News, Briefings, Features, Portraits, Press Statements, Blogs).

- Other key communication platforms (social media, image \& video hosting sites, visits).

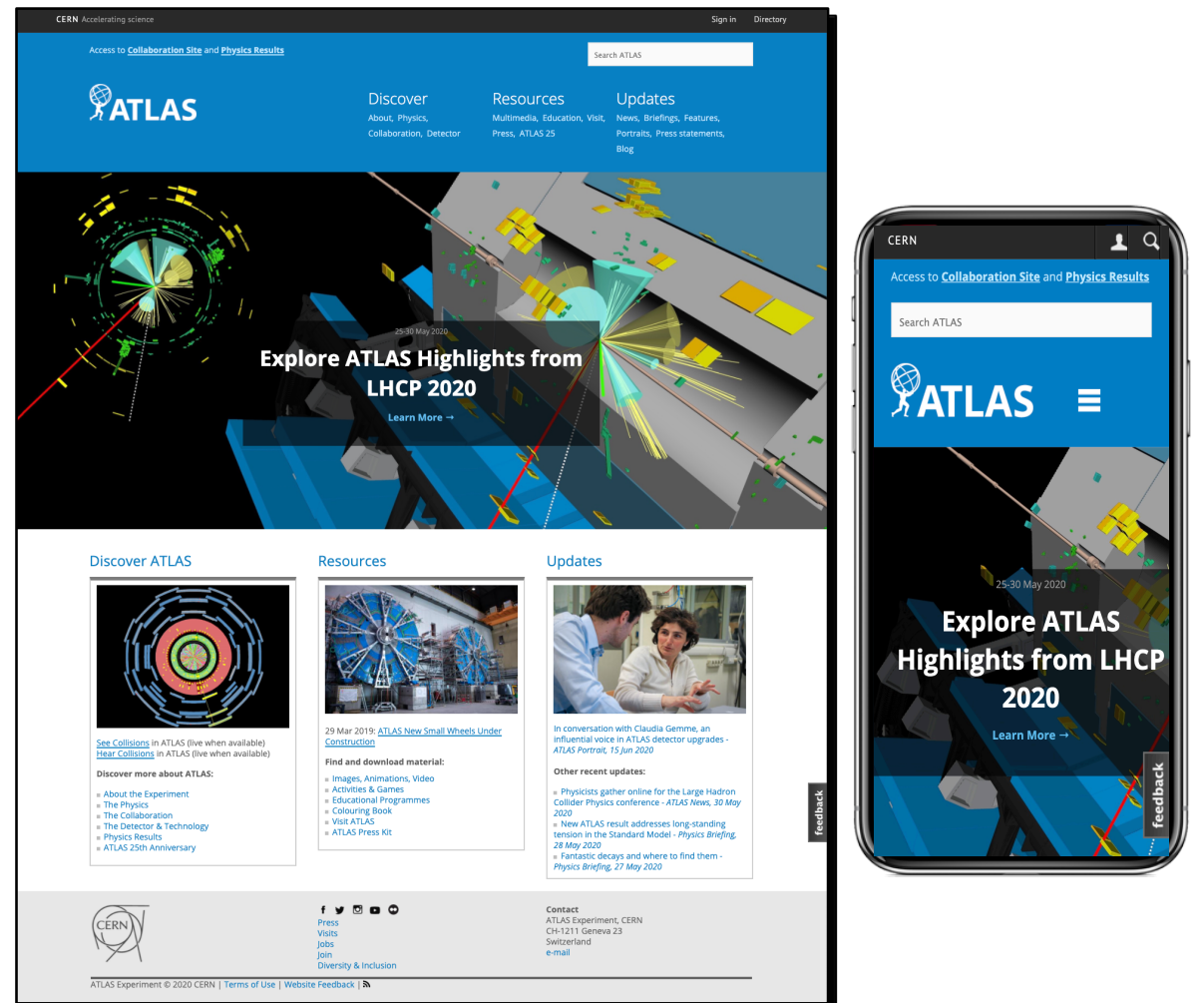

Figure 1: Welcome to the ATLAS public website on desktop or phone! Screenshots taken June 2020. 
This document describes:

- Current usage of the ATLAS public website,

- New features in the Drupal 8 version of the ATLAS public website.

\section{ATLAS public website usage}

Figure 2 presents the audience evolution of the ATLAS public website in terms of page views per week from its first publication in July 2016 through June 2020. There has been steady growth over four years with occasional spikes corresponding to key events and physics results. Current readership is around 30,000 page views per week.

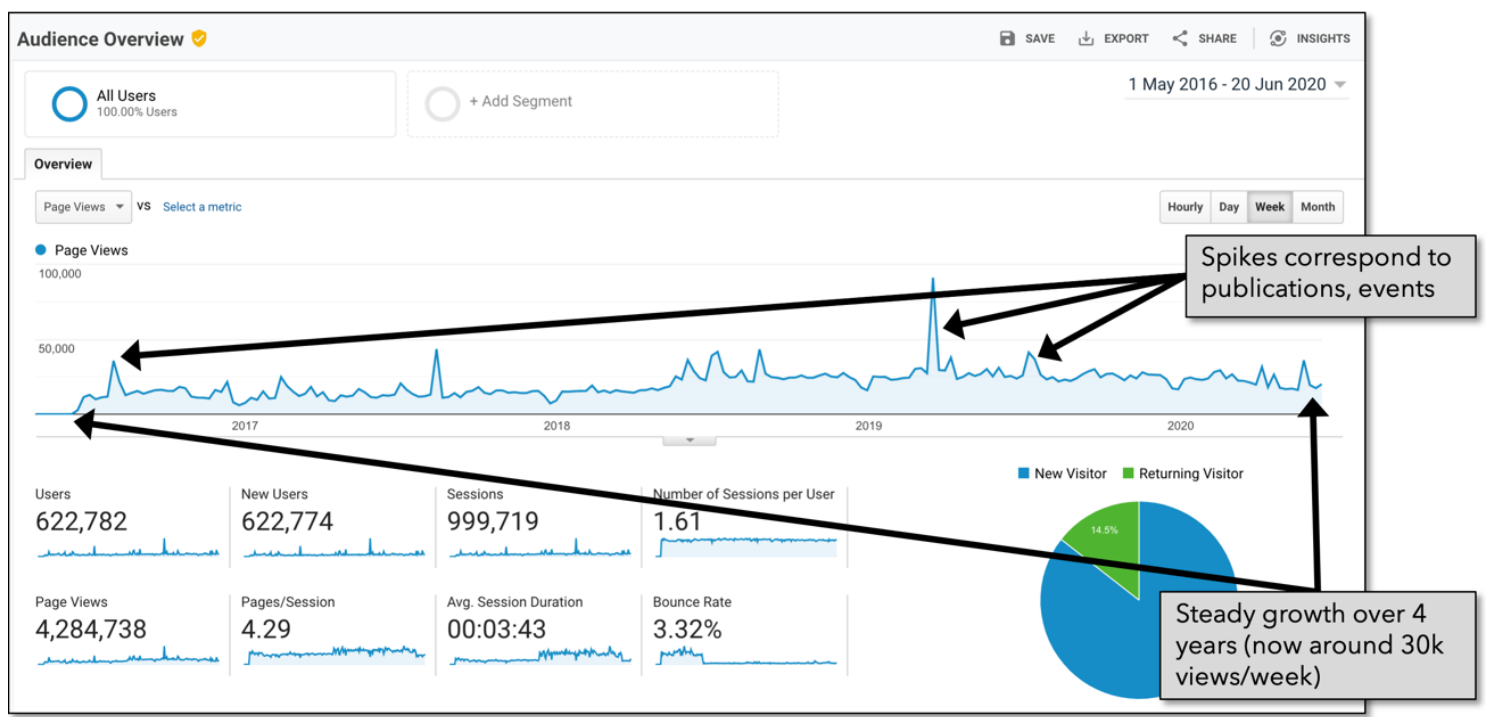

Figure 2: Visit evolution of ATLAS public website in terms of page views / week.

The current ATLAS public website was developed in version 7 of the Drupal [4] content management system, which replaced the original HTML site. CERN support for Drupal 7 ends during 2020, but support for Drupal 8 is guaranteed afterwards. This provides an opportunity to improve the ATLAS public website. One lesson learnt from the original development is that maintainabilty is key to simplify future migrations.

\section{New features in the ATLAS public website with Drupal 8}

Upgrading to Drupal 8 allows us to improve the design of the ATLAS public website, such as adding dynamic content, thus reinforcing our communication strategy. Using the "CERN Override" theme rather than an ATLAS-specific theme will allow us to reduce the number of specialised templates, thus reducing future maintenance requirements. The CERN support team has been very helpful in providing most of the functionality we need. Unfortunately, the migration is not automatic, one of the reasons being that it includes a change of template language. Figure 3 presents a potential home-page video layout in Drupal 8. This is an example of the dynamic content being prepared. 
Figure 4 presents an example page about the ATLAS Experiment. A striking picture is put at the top of the page to draw attention and accompany the text.
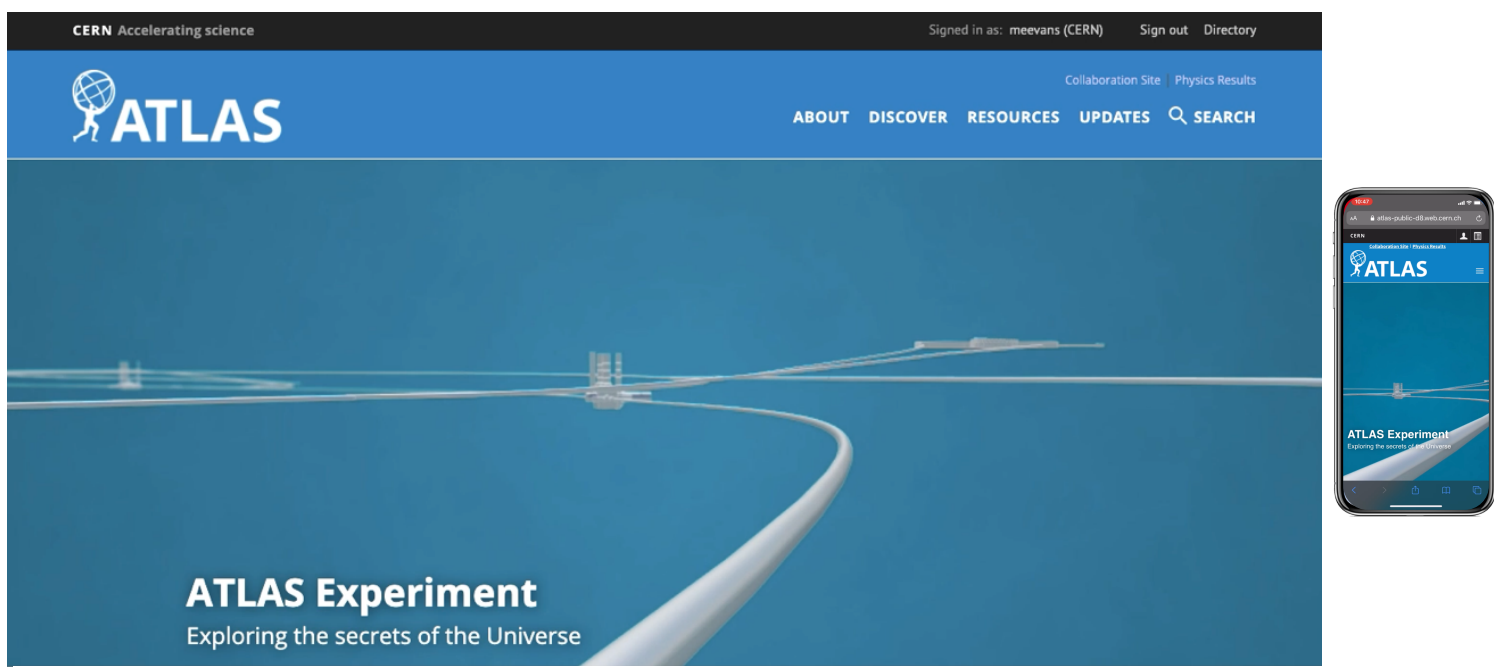

Figure 3: Potential home page video layout on desktop and phone.

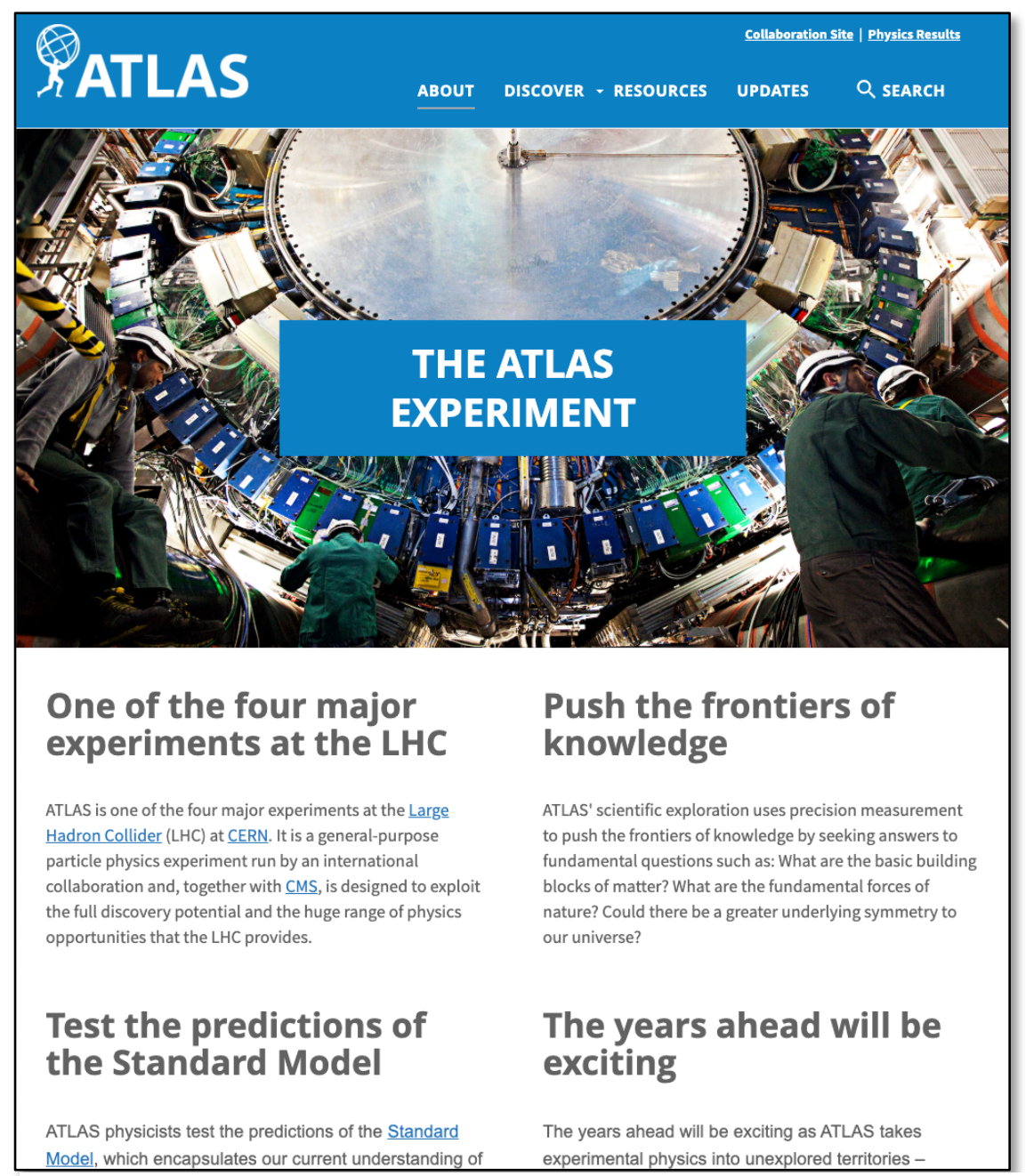

Figure 4: Potential "About" page describing the ATLAS Experiment. 
A new example of the Updates landing page is shown in Figure $\underline{5}$. This page would port nicely between different screen sizes, such as desktop, tablet and phone. Such a page would include:

1. Top Feature: Update that we want to push to the public, e.g. a new physics result.

2. Features: Updates that may have previously been top features.

3. Articles: remaining Updates, along with their blurbs.

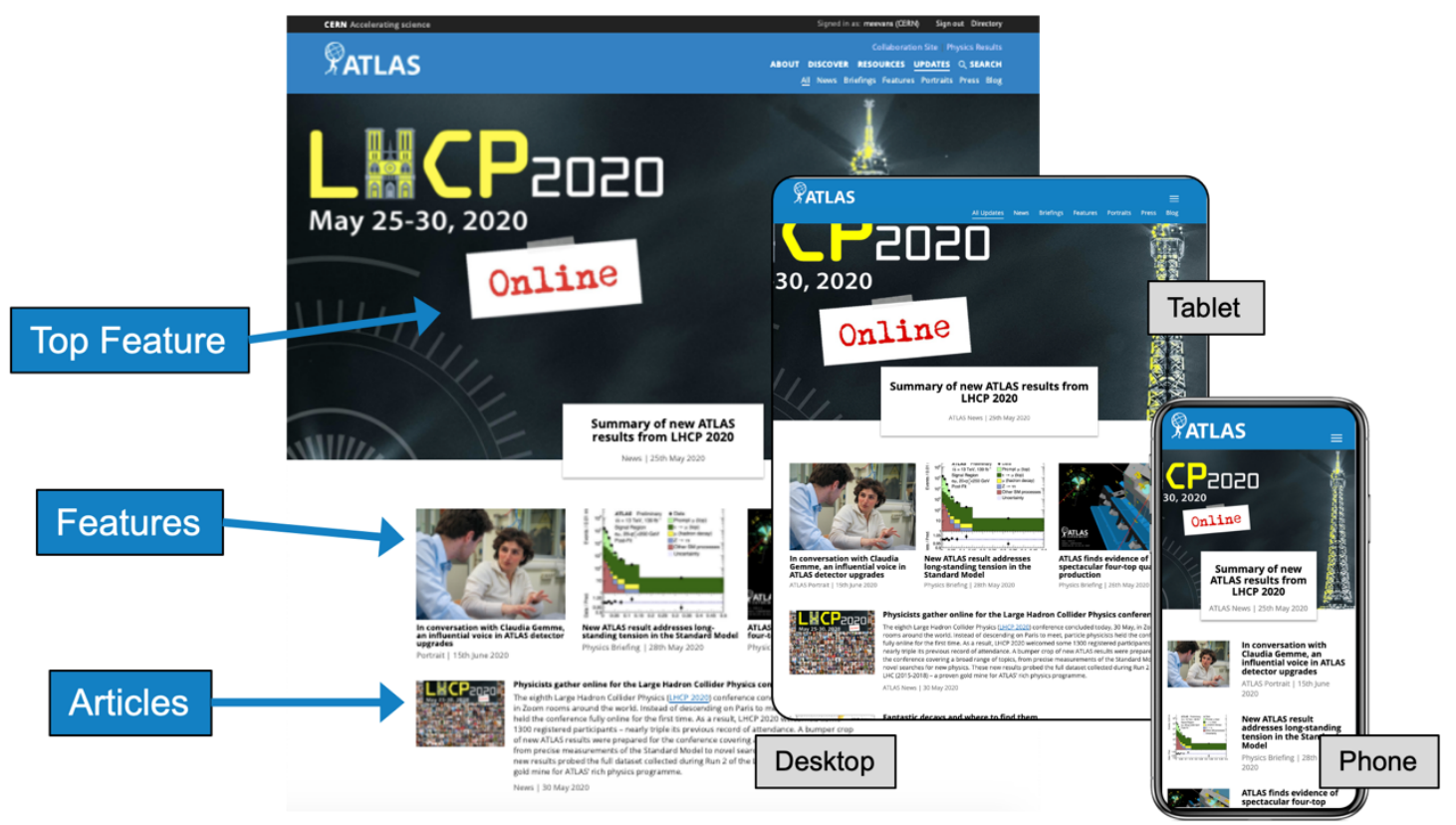

Figure 5: Potential Updates landing page for the different screen sizes of desktop, tablet and phone.

Another new feature presented in Figure $\underline{6}$ is the possibility to search through Updates using title, author or tags such as "Higgs physics". Yet another possibility is to use a new sub-menu to browse Updates.

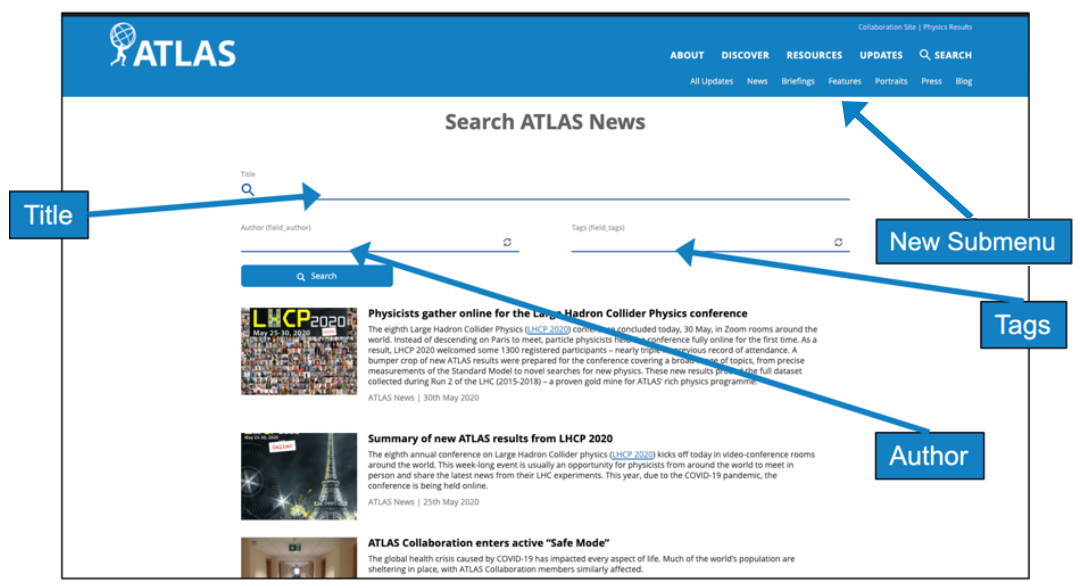

Figure 6: The new ATLAS public website will provide functionailty to search through Updates.

Also being updated are the pages highlighting both current and past management members, so that visitors can get an idea of what it means to be part of this huge collaboration. Individual 
authors who have contributed to ATLAS Updates and Blogs can also write their own pages. An individual's page would contain their picture, bio and Updates to which they have contributed. Figure 7 shows the general page for ATLAS Management and Authors pages.
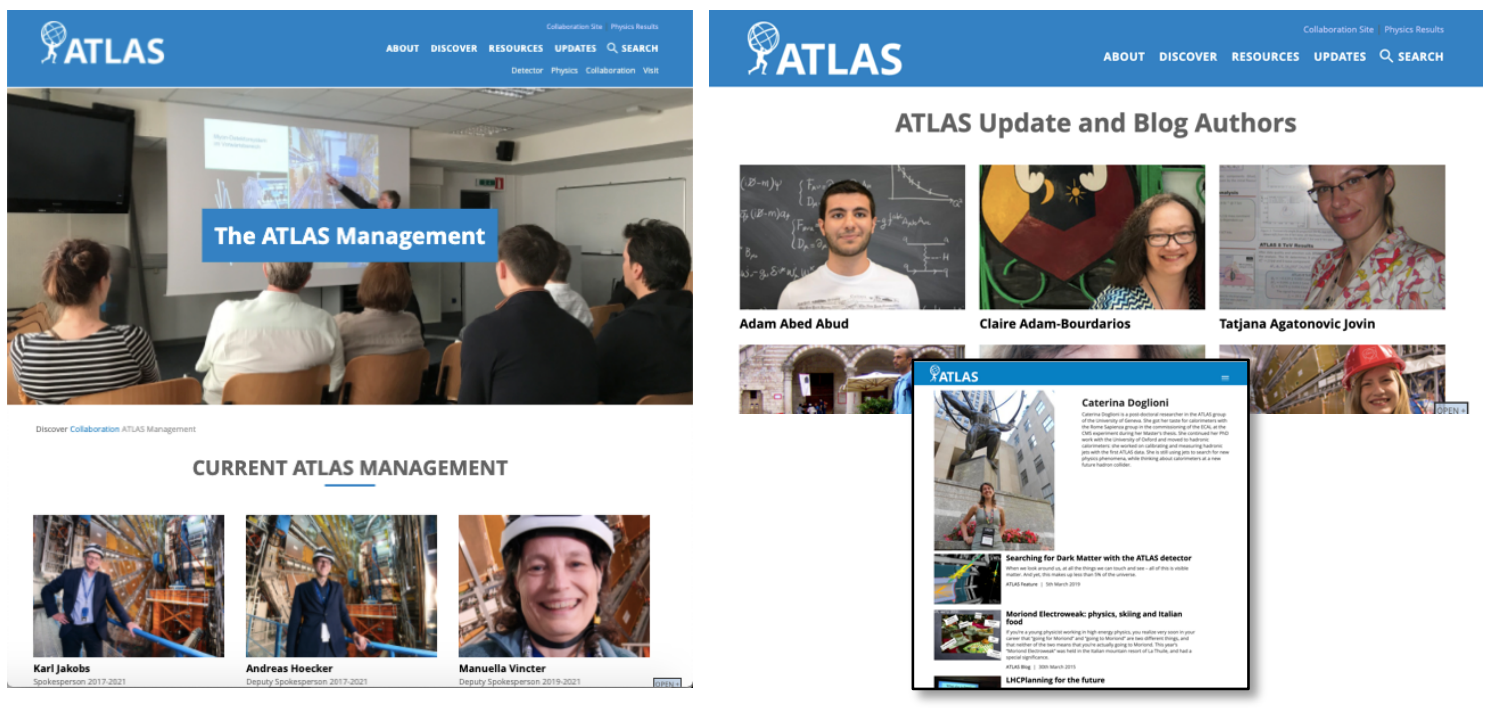

Figure 7: Potential new website pages for the ATLAS Management and Authors of ATLAS Updates.

\section{Summary}

The ATLAS Education \& Outreach group is well into the process of migrating its existing public website from version 7 to version 8 of the Drupal content management system. The team is building the new site using the CERN Override theme and is receiving significant support from the CERN web-development team. The new pages will build on four years of experience with the existing site, present more in-depth content and feature a more dynamic layout with reactive response to varying screen sizes.

This document presents the combined effort of a number of people, including the ATLAS Communication Task Leader, Web Developer, Education \& Outreach Coordinators and the author. We acknowledge our ATLAS colleagues and many others participating in the various surveys and interviews for their input and advice in the development of the website. We also thank our partners in the CERN ECO and IT groups for providing important technical support and advice. Finally, we acknowledge and thank our home institutes, for supporting our research.

\section{References}

[1] Large Hadron Collider: https://home.cern/science/accelerators/large-hadron-collider.

[2] ATLAS Public Website: https://atlas.cern.

[3] ATLAS Collaboration: JINST 3 (2008) S08003.

[4] Drupal is an Open Source Content Management System: https://www.drupal.org. 